\title{
The Impact of Personality Traits on Ratings of Obsessive-Compulsive Symptoms
}

\author{
Min Jung Huh', Geumsook Shim², Min Soo Byun', Sung Nyun Kim¹, Euitae Kim¹, \\ Joon Hwan Jang ${ }^{1}$, Min-Sup Shin ${ }^{1}$ and Jun Soo Kwon ${ }^{1,3} \bowtie$ \\ 1'Department of Psychiatry, Seoul National University College of Medicine, Seoul, Republic of Korea \\ ${ }^{2}$ Institute of Forensic Psychiatry Ministry of Justice, Gongju, Republic of Korea \\ ${ }^{3}$ Department of Brain and Cognitive Sciences - World Class University Program, College of Natural Sciences, Seoul National University, Seoul, \\ Republic of Korea
}

Objective The goal of this study was to evaluate consistencies and discrepancies between clinician-administered and self-report versions of the Yale-Brown Obsessive Compulsive Scale (Y-BOCS) and to examine relationships between these scales and personality traits. Methods A total of 106 patients with obsessive-compulsive disorder (OCD) participated in this study. All participants were assessed with both clinician-administered and self-report versions of the Y-BOCS. The Structured Clinical Interview for DSM-IV Axis II Disorders Personality Questionnaire (SCID-II-PQ) was used to evaluate relationships between personality traits and scores on the Y-BOCS.

Results Scores on the clinician-administered Y-BOCS and its obsession subscale were significantly higher than were those on the selfreport version. However, we found no significant differences in compulsion subscale scores. We also found that the discrepancies in the scores on the two versions of the Y-BOCS and its compulsion subscale were significantly positively correlated with scores for narcissistic personality traits on the SCID-II-PQ. Additionally, narcissistic personality traits had a significant effect on the discrepancy in the scores on the two versions of the Y-BOCS and its compulsion subscale in the multiple linear regression analysis.

Conclusion This is the first study to elucidate relationships between personality traits and discrepancies between scores on the two versions of the Y-BOCS. Although clinicians tend to rate obsessive symptoms as being more severe than do patients, clinicians may underestimate the degree to which individuals with narcissistic personality traits suffer more from subjective discomfort due to compulsive symptoms. Therefore, the effect of personality traits on symptom severity should be considered in the treatment of OCD.

Psychiatry Investig 2013;10:259-265

Key Words Obsessive-compulsive disorder, Yale-Brown Obsessive Compulsive Scale, SCID-II personality questionnaire, Personality trait.

\section{INTRODUCTION}

Patients with obsessive-compulsive disorder (OCD) suffer from recurrent obsessions and compulsions that cause marked distress or impaired functioning. The Yale-Brown Obsessive Compulsive Scale (Y-BOCS) is the most widely used instrument for assessing these symptoms. ${ }^{1,2}$ The full version of the Y-BOCS is administered by a well-trained clinician during an

Received: July 1, 2012 Revised: March 23, 2013

Accepted: April 2, 2013 Available online: September 16, 2013

$\triangle$ Correspondence: Jun Soo Kwon, MD, PhD

Department of Psychiatry, Seoul National University College of Medicine, 101 Daehak-ro, Jongno-gu, Seoul 110-744, Republic of Korea

Tel: +82-2-2072-2450, Fax: +82-2-747-9063, E-mail: kwonjs@snu.ac.kr

(a) This is an Open Access article distributed under the terms of the Creative Commons Attribution Non-Commercial License (http://creativecommons.org/licenses/by$\mathrm{nc} / 3.0$ ) which permits unrestricted non-commercial use, distribution, and reproduction in any medium, provided the original work is properly cited. interview that takes about 30 minutes. As the clinician-administered version can be time-consuming, a self-report version of the Y-BOCS was developed by Baer in 1991. ${ }^{3}$ Previous studies have compared the clinician-administered Y-BOCS with the self-report version ${ }^{4,5}$ and found significant correlations between individual items on the two scales ( $\mathrm{r}=0.41$ to 0.76 ) among 36 OCD patients. ${ }^{4} \mathrm{~A}$ more recent study with a larger sample also found that the total Y-BOCS scores on the two scales showed significant intraclass correlations (ICCs) (ICC $=0.64$ ) in 86 patients with OCD. 5 They also found that the mean score on the clinician-administered Y-BOCS was significantly higher than that on the self-report version of the Y-BOCS.

However, no previous study has elucidated the factors related to discrepancies between the two versions of this instrument. In the assessment of depression, discrepancies between scores on the clinician-rated Hamilton Depression Rating Scale and 
the self-report Beck Depression Inventory have been associated with high neuroticism and low extraversion on the NEOFive-Factor Inventory, a personality inventory. ${ }^{6}$ Although many studies have explored comorbid axis II personality disorders in OCD patients, ${ }^{7-10}$ no previous research has investigated the personality traits related to discrepancies between self-reported and clinician-administered measures of obsessive-compulsive symptoms. Despite variations in results regarding comorbid personality disorders, it is well known that patients with OCD have high rates of comorbidity with cluster-C personality disorders. Additionally, some studies have found that comorbid cluster-B personality disorders are associated with poor treatment response. ${ }^{11,12}$ Nonetheless, no previous study has examined relationships between personality traits and discrepancies between self-reports and clinician ratings. This study examined consistencies and discrepancies between the two versions of the Y-BOCS and elucidated the personality traits that affect such discrepancies.

\section{METHODS}

\section{Participants}

Patients were recruited from the OCD outpatient clinic at Seoul National University Hospital (SNUH) during 2006-2011. A total of 106 patients met DSM-IV criteria for obsessive-compulsive disorder (OCD).

None of the participants was taking medicine at intake; 48 patients were drug naïve, and 58 had been unmedicated for at least 4 weeks. No patient had previously received cognitivebehavioral therapy. The sample consisted of 64 males and 42 females, with a mean age of $26.75 \pm 8.31$ years. The mean age of onset and the mean duration of illness were 19.33 \pm 7.91 and $7.44 \pm 6.50$ years, respectively. The mean duration of participants' education was $14.23 \pm 2.53$ years. Participants suffered from the following comorbid axis I psychiatric disorders: mood disor$\operatorname{der}(n=22)$, general anxiety disorder $(n=4)$, tic disorder $(n=2)$, impulse-control disorder $(\mathrm{n}=1)$, and somatoform disorder $(\mathrm{n}=1)$. Participants who were diagnosed with schizophrenia or mental retardation were excluded. Participants with a history of significant neurologic or medical illness were also excluded. This study was approved by the Institutional Review Board of SNUH.

\section{Clinical measures}

Yale-Brown Obsessive Compulsive Scale (Y-BOCS)

We used the Yale-Brown Obsessive Compulsive Scale (YBOCS) to assess the severity of obsessions and compulsions. Symptom severity was measured in five domains: time spent, interference, distress, efforts to resist, and perceived control. Each domain was rated on a five-point Likert scale from 0 (not at all/none) to 4 (extreme).

All participants were assessed with the clinician-administered and self-report versions of the Y-BOCS. ${ }^{13}$ The clinicianadministered Y-BOCS was administered by psychiatrists who worked at the OCD outpatient clinic at SNUH during 20062011. However, the therapist for the subjects did not participate in the Y-BOCS rating. Although we did not assess interrater reliability, all raters were clinically well-trained boardcertified psychiatrists who had sufficient experience with the Y-BOCS. Most patients (90.6\%) were assessed with the clinician-administered Y-BOCS prior to completing the self-report Y-BOCS. Only $9.4 \%$ of the patients completed the selfreport version of the Y-BOCS first. The mean time between completion of the two versions of the Y-BOCS was 8.0 days.

\section{Structured Clinical Interview for DSM-IV Axis II Disorders Personality Questionnaire (SCID-II-PQ)}

Personality traits were assessed with the Korean version of the SCID-II-PQ, which consists of 119 items rated on a dichotomous (yes/no) scale. ${ }^{14}$ This study used the SCID-II-PQ as a screening tool given the high level of agreement (kappa=0.78) between the SCID-II clinical interview and the SCID-II personality questionnaire after adjusting the cut-off value. ${ }^{15}$

\section{Beck Depression Inventory (BDI) and \\ Beck Anxiety Inventory (BAI)}

In this study, the self-report $\mathrm{BDI}^{16}$ and $\mathrm{BAI}^{17}$ were used to assess the severity of depressive and anxiety symptoms in patients with OCD. Mean scores on the BDI and BAI were 17.42 \pm 9.69 and $18.09 \pm 12.08$, respectively. Of the 106 patients, two patients did not completed the BDI and BAI $(n=104)$.

\section{Statistical analysis}

To investigate consistencies and discrepancies between scores

Table 1. Difference between the self-report and clinician-administered version of $Y-B O C S(N=106)$

\begin{tabular}{|c|c|c|c|c|}
\hline \multirow{2}{*}{ Scale } & \multicolumn{2}{|c|}{ Mean \pm SD } & \multirow{2}{*}{$\mathrm{t}$} & \multirow{2}{*}{ p-value } \\
\hline & Self-report & Clinician-administered & & \\
\hline Total Y-BOCS score & $23.7 \pm 7.1$ & $25.6 \pm 6.5$ & 2.13 & $0.034^{*}$ \\
\hline Obsessions subscale & $12.3 \pm 3.4$ & $13.8 \pm 3.1$ & 3.26 & $0.001^{*}$ \\
\hline Compulsions subscale & $11.3 \pm 4.4$ & $11.9 \pm 4.5$ & 0.90 & 0.372 \\
\hline
\end{tabular}

${ }^{*} \mathrm{p}<0.05$. Y-BOCS: Yale-Brown Obsessive Compulsive Scale 
on the two versions of the Y-BOCS, we used independent ttests between means and calculated the intraclass correlation coefficients (ICCs) for the correlation analyses. We calculated discrepancy scores by subtracting the scores on the clinicianadministered Y-BOCS from those on the self-report Y-BOCS. ${ }^{6}$ Multiple linear regression analysis was conducted to investigate whether personality traits were associated with discrepancies. Statistical analyses were performed using Predictive Analytics Software (PASW) version 18.0 (SPSS, Inc., Chicago, USA).

\section{RESULTS}

\section{Consistencies and discrepancies between scores on clinician-administered and self-report versions of the Y-BOCS}

The mean scores on the clinician-administered and self-report versions of the Y-BOCS are shown in Table 1. Independent $t$-tests revealed that scores on the clinician-administered Y-BOCS and on the obsession subscale were significantly higher than were those on the self-report versions. However, the difference between scores on the two versions of the compulsion subscale was not significant $(\mathrm{p}=0.372)$.

To assess the strength of the correlations, we calculated the ICC between the two versions of the Y-BOCS. We found significant correlations between scores on the two versions of the Y-BOCS and the obsession and compulsion subscales. However, a lower ICC was found for the obsession subscale (ICC= $0.61, \mathrm{p}<0.0001)$ than for the total Y-BOCS (ICC $=0.70, \mathrm{p}<$ 0.0001 ) and the compulsion subscale (ICC $=0.68, \mathrm{p}<0.0001$ ).

Table 2 presents the correlations between the scores on the individual items in the two versions of the Y-BOCS. Although significant correlations between scores on individual items were found, the correlations between the two versions of items addressing "efforts to resist obsessions" and "efforts to resist compulsions" were lower than were others ( $\mathrm{r}=0.44$ for item 4 on the obsession subscale, $r=0.44$ for item 9 on the compulsion subscale).

The internal consistency was high for both versions of the Y-BOCS. The Cronbach's alphas for the self-report Y-BOCS were 0.88 (total), 0.78 (obsession subscale), and 0.85 (compulsion subscale). Those for the clinician-administered Y-BOCS were 0.84 (total), 0.68 (obsession subscale), and 0.88 (compulsion subscale).

Total Y-BOCS scores can be classified into five groups according to severity: subclinical (0-7), mild (8-15), moderate (16-23), severe (24-31), and extreme (32-40). To assess the degree of consistency in this categorical classification, we calculated the value of the weighted kappa and found a moderate degree of agreement between the two scales (weighted kappa=0.43).

\section{Relationships between Y-BOCS and SCID-II-PQ scores}

We also investigated the relationship between symptom severity on the Y-BOCS and personality traits. The data indicated that the most common comorbid personality traits were obsessive-compulsive (71.70\%), borderline (60.38\%), narcissistic (55.66\%), and avoidant (51.89\%) (Table 3).

In addition, we examined correlations between SCID-II personality questionnaire scores and two versions of Y-BOCS scores (total scores, compulsion subscale scores and obsession subscale scores) (Table 4). The significant positive correlations were observed in cluster $\mathrm{A}$ and cluster $\mathrm{C}$ personality traits

Table 2. Individual item correlations between two versions of $Y$ BOCS $(\mathrm{N}=106)$

\begin{tabular}{lcc}
\hline \multirow{2}{*}{ Individual item } & \multicolumn{2}{c}{ Correlation across scale versions } \\
\cline { 2 - 3 } & $\mathrm{r}$ & $\mathrm{p}$-value \\
\hline Obsessions subscale & 0.57 & $<0.0001^{*}$ \\
Time spent & 0.47 & $<0.0001^{*}$ \\
Interference & 0.50 & $<0.0001^{*}$ \\
Distress & 0.44 & $<0.0001^{*}$ \\
Efforts to resist & 0.49 & $<0.0001^{*}$ \\
Perceived control & & \\
Compulsions subscale & 0.59 & $<0.0001^{*}$ \\
Time spent & 0.56 & $<0.0001^{*}$ \\
Interference & 0.63 & $<0.0001^{*}$ \\
Distress & 0.44 & $<0.0001^{*}$ \\
Efforts to resist & 0.51 & $<0.0001^{*}$ \\
Perceived control &
\end{tabular}

${ }^{*} \mathrm{p}<0.05$. Y-BOCS: Yale-Brown Obsessive Compulsive Scale

Table 3. Frequency of personality traits found in OCD patients $(\mathrm{N}=106)$

\begin{tabular}{lccc}
\hline \multicolumn{1}{c}{ SCID-II-PQ } & Cutoff & $\mathrm{N}$ & $\%$ \\
\hline Avoidant & 4 & 55 & 51.89 \\
Dependent & 5 & 22 & 20.75 \\
Obsessive-compulsive & 4 & 76 & 71.70 \\
Passive-aggressive & 4 & 41 & 38.68 \\
Depressive & 5 & 42 & 39.62 \\
Paranoid & 4 & 51 & 48.11 \\
Schizotypal & 5 & 20 & 18.87 \\
Schizoid & 4 & 9 & 8.49 \\
Histrionic & 5 & 9 & 8.49 \\
Narcissistic & 5 & 59 & 55.66 \\
Borderline & 5 & 64 & 60.38 \\
Antisocial & 3 & 22 & 20.75 \\
\hline OCD: obsessive-compusive & disorder, SCID-II-PQ: Structured
\end{tabular}

OCD: obsessive-compulsive disorder, SCID-II-PQ: Structured Clinical Interview for DSM-IV Axis II Disorders Personality Questionnaire 
Table 4. Correlations between the SCID-II-PQ scores and the $\mathrm{Y}$-BOCS scores $(\mathrm{N}=106)$

\begin{tabular}{|c|c|c|c|c|c|c|}
\hline \multirow{3}{*}{ SCID-II-PQ scores } & \multicolumn{6}{|c|}{ Y-BOCS scores } \\
\hline & \multicolumn{3}{|c|}{ Self-report } & \multicolumn{3}{|c|}{ Clinician-administered } \\
\hline & Stot & Scom & Sobs & Ctot & Ccom & Cobs \\
\hline Avoidant & $0.27 * *$ & $0.26^{* *}$ & $0.23 *$ & $0.24^{*}$ & 0.15 & $0.29 * *$ \\
\hline Dependent & $0.20 *$ & $0.20^{*}$ & 0.17 & $0.20^{*}$ & 0.09 & $0.29^{* *}$ \\
\hline Obsessive-compulsive & $0.20^{*}$ & $0.20 *$ & 0.15 & $0.24^{*}$ & $0.21^{*}$ & $0.21^{*}$ \\
\hline Passive-aggressive & 0.18 & 0.15 & 0.18 & 0.10 & 0.03 & 0.15 \\
\hline Depressive & $0.28^{* *}$ & $0.22 *$ & $0.31^{* *}$ & $0.23 *$ & 0.11 & $0.31^{* *}$ \\
\hline Paranoid & $0.25^{*}$ & $0.22 *$ & $0.23 *$ & $0.21^{*}$ & 0.15 & $0.22 *$ \\
\hline Schizotypal & $0.22 *$ & $0.20^{*}$ & $0.20^{*}$ & $0.19 *$ & 0.15 & 0.19 \\
\hline Schizoid & $0.23^{*}$ & $0.22 *$ & 0.19 & 0.19 & 0.15 & 0.17 \\
\hline Histrionic & -0.03 & -0.03 & -0.03 & -0.13 & -0.10 & -0.11 \\
\hline Narcissistic & 0.12 & 0.11 & 0.11 & -0.07 & -0.07 & -0.05 \\
\hline Borderline & 0.19 & 0.18 & 0.17 & 0.09 & 0.05 & 0.12 \\
\hline Antisocial & 0.01 & 0.04 & -0.03 & -0.17 & -0.12 & -0.18 \\
\hline
\end{tabular}

${ }^{*} \mathrm{p}<0.05,{ }^{* *} \mathrm{p}<0.01$. Y-BOCS: Yale-Brown Obsessive Compulsive Scale, SCID-II-PQ: Structured Clinical Interview for DSM-IV Axis II Disorders Personality Questionnaire, Stot: total Y-BOCS scores in self-report version, Scom: compulsion subscale scores in self-report version, Sobs: obsession subscale scores in self-report version, Ctot: total Y-BOCS in clinician-administered version, Ccom: compulsion subscale scores in clinician-administered version, Cobs: obsession subscale scores in clinician-administered version

Table 5. Correlations between the SCID-II-PQ scores and the discrepancy scores $(\mathrm{N}=106)$

\begin{tabular}{lccc}
\hline \multirow{2}{*}{ SCID-II-PQ scores } & \multicolumn{3}{c}{ Discrepancy scores } \\
\cline { 2 - 4 } & Dtot & Dcom & Dobs \\
\hline Avoidant & 0.07 & 0.13 & -0.04 \\
Dependent & 0.02 & 0.13 & -0.12 \\
Obsessive-compulsive & -0.03 & -0.01 & -0.05 \\
Passive-aggressive & 0.13 & 0.14 & 0.05 \\
Depressive & 0.10 & 0.13 & 0.03 \\
Paranoid & 0.07 & 0.08 & 0.03 \\
Schizotypal & 0.06 & 0.06 & 0.02 \\
Schizoid & 0.08 & 0.08 & 0.04 \\
Histrionic & 0.11 & 0.09 & 0.09 \\
Narcissistic & $0.25^{* *}$ & $0.23^{*}$ & 0.18 \\
Borderline & 0.14 & 0.15 & 0.07 \\
Antisocial & $0.22^{*}$ & $0.20^{*}$ & 0.16 \\
\hline
\end{tabular}

${ }^{*} \mathrm{p}<0.05,{ }^{* *} \mathrm{p}<0.01$. Dtot $=[$ self-report total YBOCS score]-[clinician administered total YBOCS score], Dcom=[self-report compulsion subscale score]-[clinician administered compulsion subscale score], Dobs $=[$ self-report obsession subscale score-clinician administered obsession subscale score]. SCID-II-PQ: Structured Clinical Interview for DSM-IV Axis II Disorders Personality Questionnaire

with the self-reported total Y-BOCS scores $(\mathrm{r}=0.27, \mathrm{p}<0.01$ for avoidant trait, $\mathrm{r}=0.25$ for paranoid trait, $\mathrm{p}<0.05)$. This suggests that the obsessive-compulsive symptom severity is correlated with these personality traits. However, there were no significant correlations between the cluster $\mathrm{B}$ personality traits and the Y-BOCS scores.
To elucidate the relationship of discrepancies between the self-report and clinician-administered scores with personality traits, we calculated discrepancy scores by subtracting clinicianadministered Y-BOCS scores from self-report Y-BOCS scores (Table 5); thus, higher discrepancy scores reflected higher scores on the self-report than on the clinician-administered YBOCS, indicating that patients rated their symptoms as more severe than did clinicians. Table 5 shows that the discrepancies between total Y-BOCS scores (Dtot) were positively correlated with narcissistic $(\mathrm{r}=0.25, \mathrm{p}<0.01)$ and antisocial $(\mathrm{r}=$ $0.22, p<0.05)$ personality traits. Furthermore, discrepancies on the compulsion subscale (Dcom) were also positively correlated the narcissistic $(r=0.23, p<0.05)$ and antisocial $(r=$ $0.20, p<0.05)$ personality traits. However, we found no significant correlations between scores on the personality questionnaire and discrepancies between the two scores on the obsession subscale (Dobs). To exclude the effect of axis I comorbid conditions, such as depressive and other anxiety disorders, we conducted an additional analysis among OCD patients without comorbid axis I disorders $(\mathrm{n}=80)$. Comparisons of the mean scores on the Y-BOCS and the discrepancy scores for all patients with $\mathrm{OCD}(\mathrm{n}=106)$ with that for patients without any axis I comorbid disorders $(\mathrm{n}=80)$ revealed no significant differences between the two groups. We also observed significant positive correlations between narcissistic personality traits and discrepancies in total Y-BOCS ( $\mathrm{r}=0.27, \mathrm{p}=.014)$ and compulsion subscale $(r=0.23, p=0.044)$ scores in patients with $\mathrm{OCD}$ but without comorbid conditions $(\mathrm{n}=80)$. However, we found 
Table 6. Multiple linear regression analysis $(\mathrm{N}=104)$

\begin{tabular}{|c|c|c|c|c|c|c|}
\hline \multirow{2}{*}{ SCID-II-PQ scores } & \multicolumn{3}{|c|}{ Dtot } & \multicolumn{3}{|c|}{ Dcom } \\
\hline & $\beta$ & $\mathrm{t}$ & $\mathrm{p}$ & $\beta$ & $\mathrm{t}$ & $\mathrm{p}$ \\
\hline Narcissistic & 0.246 & 2.526 & $0.013^{*}$ & 0.220 & 2.263 & $0.026^{*}$ \\
\hline BDI & 0.167 & 1.37 & 0.174 & 0.202 & 1.657 & 0.101 \\
\hline \multirow[t]{3}{*}{ BAI } & -0.026 & -0.209 & 0.835 & -0.023 & -0.184 & 0.855 \\
\hline & \multicolumn{3}{|c|}{$\mathrm{R}=0.295, \mathrm{R}^{2}=0.087$} & \multicolumn{3}{|c|}{$\mathrm{R}=0.297, \mathrm{R}^{2}=0.088$} \\
\hline & \multicolumn{3}{|c|}{$\mathrm{F}=3.178, \mathrm{p}=0.027$} & \multicolumn{3}{|c|}{$\mathrm{F}=3.230, \mathrm{p}=0.026$} \\
\hline
\end{tabular}

${ }^{*} \mathrm{p}<0.05$. Dtot $=$ [self-report total YBOCS score]-[clinician administered total YBOCS score], Dcom=[self-report compulsion subscale score][clinician administered compulsion subscale score]. BDI: Beck Depression Inventory, BAI: Beck Anxiety Inventory, SCID-II-PQ: Structured Clinical Interview for DSM-IV Axis II Disorders Personality Questionnaire

no significant positive correlations between antisocial personality traits and discrepancies between the scores on the two versions of the Y-BOCS when patients with OCD with axis I comorbid conditions were excluded, suggesting that the correlations between antisocial personality traits and discrepancy scores may have been influenced by the inclusion of patients with comorbid axis I disorders in our OCD sample.

Furthermore, narcissistic personality traits had significant effects on the discrepancies between scores on the two versions of both the full Y-BOCS and the compulsion subscale when scores on the self-report BDI and BAI were controlled in multiple linear regression analysis (Table 6).

\section{DISCUSSION}

In this study, we found that mean scores on the clinicianadministered Y-BOCS were significantly higher than were those on the self-report version. The main difference between the two versions of the Y-BOCS was attributable to the obsession subscale, which was the only subscale on which scores on the two versions differed significantly. This implies that clinicians tend to rate obsessive symptoms as more severe than do patients. The mean scores on the two compulsion subscales did not differ significantly. There was a previous report which found that scores on the clinician-rated Y-BOCS were higher than the self-rated scores. ${ }^{5}$ However, the significant differences were found between scores on the compulsion subscale not on the obsession subscale. ${ }^{5}$ These results can be interpreted in several ways. First, we believe that compulsions are easier to rate than are obsessions, which may be due to the characteristics of the two phenomena. Indeed, obsessions are more subjective and difficult to rate, whereas compulsions can be more objective and easier to rate, which may reduce discrepancies between the two compulsion scores. Furthermore, the ICCs for the obsession subscales (ICC $=0.61$ ) were lower than were those for the compulsion subscales (ICC $=0.68$ ), which also suggests a greater discrepancy between the obsession than between the compulsion subscales.
With regard to the comparisons between individual items on the two versions of the Y-BOCS, the Pearson's correlation coefficients for all items ranged from $r=0.44$ to $r=0.63$ ( $p<$ 0.0001 ) (Table 2). Items addressing "efforts to resist obsessions" (item 4) and "efforts to resist compulsions" (item 9) had the lowest value ( $\mathrm{r}=0.44)$, which is consistent with the results of a previous study in which "efforts to resist obsessions" also had the lowest value $(\mathrm{r}=0.26){ }^{5}$ The mean scores of these items in the clinician-rated Y-BOCS $(2.09 \pm 1.23$ for item 4 and $2.04 \pm$ 1.23 for item 9) were higher than were those on the self-report version ( $1.61 \pm 0.92$ for item 4 and $1.79 \pm 1.13$ for item 9), suggesting that clinicians underestimate the efforts expended by patients to resist these symptoms.

Next, we investigated relationships between Y-BOCS scores and personality traits. According to our data, the most common comorbid personality traits in patients with OCD were obsessive-compulsive (71.70\%), borderline (60.38\%), narcissistic (55.66\%), and avoidant (51.89\%) (Table 3). It has been established that cluster-C personality traits are common in patients with OCD. ${ }^{7-9}$ It is noteworthy that the present study found that cluster-B personality traits (borderline and narcissistic traits) were common in patients with OCD. This result is consistent with those of a previous study, which found that OCD patients were more likely to have comorbid borderline personality disorder than were normal controls. ${ }^{18}$ Additionally, another study found that patients with OCD reported having $22 \%$ of the narcissistic and $14 \%$ of the borderline personality traits included in the SCID-II screening questionnaire. ${ }^{9}$

One of the common features of cluster-B personality disorders is impulsivity. Indeed, the diagnostic criteria for borderline personality disorder include impulsivity that is potentially self-damaging (e.g., substance abuse, reckless driving, binge eating). OCD is located at the compulsive end of the compulsive-impulsive spectrum, whereas borderline personality disorder is located at the impulsive end. Because compulsivity and impulsivity both involve repetitive behaviors and the inability to delay or inhibit these behaviors, there are substantial areas of overlap between OCD and borderline per- 
sonality disorder. ${ }^{19}$ Additionally, both the compulsiveness in $\mathrm{OCD}$ and the impulsiveness in cluster-B personality traits involve an increase in tension before enactment of the repetitive behavior, which is followed by a relief of anxiety. ${ }^{20}$

Furthermore, we found that the discrepancy between selfreported and clinician-rated Y-BOCS scores were positively correlated with scores for narcissistic personality traits (Table 5). This implies that the suffering of patients with the narcissistic personality traits may be underestimated by clinicians. Additionally, we also found positive correlations between the discrepancy between the compulsion subscales (Dcom) and scores for narcissistic personality traits. These data suggest that subjective discomfort due to compulsive symptoms may be underestimated by clinicians when scores for narcissistic personality traits are high. The definition of narcissism includes increased interest in one's self. ${ }^{21}$ Thus, patients with OCD who have narcissistic personality traits may rate their symptoms to be more severe than do clinicians. Additionally, cluster-B personality traits have dramatic, emotional, or erratic qualities, ${ }^{21}$ which may result in a greater discrepancy in the two versions of the compulsion subscales. According to the regression analysis, narcissistic personality traits had a significant effect on discrepancies between scores on the two versions of the YBOCS and of the compulsion subscale. This finding suggests that, among the cluster B-personality traits, narcissistic traits are significantly associated with discrepancy scores.

As this research was an exploratory investigation of the possible relationship between Y-BOCS scores and personality traits, correlation analyses were conducted using an alpha value of 0.05 as the threshold for statistical significance. When Bonferroni corrections were performed for the correlation analysis between self-reported/clinician-administered Y-BOCS scores and personality traits, only the Pearson's correlation coefficient for the relationship between depressive traits and Sobs $(\mathrm{r}=0.31, \mathrm{p}=0.001)$ and Cobs $(\mathrm{r}=0.31, \mathrm{p}=0.001)$ remained significant $(\mathrm{p}<0.0028)$. Furthermore, the Pearson's correlation coefficients between discrepancy scores and narcissistic or antisocial traits were not significant after Bonferroni correction. However, these results may provide clues about potential predictors of the discrepancy scores in the multiple regression analysis.

The analysis of personality traits performed in this study was limited by the fact that no SCID-II clinical interviews were conducted. It has been reported that more patients are found to suffer from a personality disorder when the SCID-II screening questionnaire (73\%) than when the SCID-II interview (54\%) is used. ${ }^{14}$ However, when the threshold for diagnosis was adjusted, the rates approached identity: $58 \%$ and $54 \%$, respectively. ${ }^{14}$ Thus, although the SCID-II personality questionnaire cannot itself determine whether patients have a personality disor- der, it can nonetheless be a useful and convenient tool for screening personality traits in clinical settings.

This study found relatively higher frequencies of all types of personality traits than did previous studies. ${ }^{9,22}$ This may have resulted from the different clinical characteristics of participants in different studies. Additionally, one previous study found that patients with OCD no longer met criteria for a personality disorder after 4 months of treatment, ${ }^{23}$ suggesting that treatment may affect a comorbid personality disorder as well as the severity of OCD itself. Previous studies that found lower rates of each type of personality trait than did our study included medicated patients with OCD who were clinically improved. ${ }^{9}$ However, our study included patients with OCD who had no history of treatment for their life or at least one month at the time of assessment, suggesting that patients with more severe psychopathology may have been included in our sample. Thus, differences in the clinical characteristics of samples may have contributed to the different rates of comorbid personality disorder among studies. ${ }^{23}$

In conclusion, this is the first study to elucidate relationships between personality traits and discrepancies in self-reported and clinician-assessed ratings of obsessive-compulsive symptoms. Our investigation of the discrepancies between the two versions of Y-BOCS (clinician-administered and self-report) suggests that clinicians tend to rate obsessive symptoms as being more severe than do patients. However, clinicians may underestimate the subjective discomfort due to compulsive symptoms experienced by patients with narcissistic personality traits. Therefore, clinicians should consider the effect of personality traits on symptom severity in the treatment of OCD. Further research using SICD-II interviews to examine relationships between personality traits and Y-BOCS scores should be conducted in the future.

\section{Acknowledgments}

This research was supported by the Mid-career Research Program through a National Research Foundation grant (20110015639).

\section{REFERENCES}

1. Goodman WK, Price LH, Rasmussen SA, Mazure C, Fleischmann RL, Hill CL, et al. The Yale-Brown Obsessive Compulsive Scale. I. Development, use, and reliability. Arch Gen Psychiatry 1989;46:1006-1011.

2. Goodman WK, Price LH, Rasmussen SA, Mazure C, Delgado P, Heninger GR, et al. The Yale-Brown Obsessive Compulsive Scale. II. Validity. Arch Gen Psychiatry 1989;46:1012-1016.

3. Baer L. Getting Control: Overcoming Your Obsessions and Compulsions. NewYork: Plume; 1991.

4. Steketee G, Frost R, Bogart K. The Yale-Brown Obsessive Compulsive Scale: interview versus self-report. Behav Res Ther 1996;34:675-684.

5. Federici A, Summerfeldt LJ, Harrington JL, McCabed RE, Purdon CL, Rowa K, et al. Consistency between self-report and clinician-administered versions of the Yale-Brown Obsessive-Compulsive Scale. J Anxiety Disord 2010;24:729-733.

6. Enns MW, Larsen DK, Cox BJ. Discrepancies between self and ob- 
server ratings of depression. The relationship to demographic, clinical and personality variables. J Affect Disord 2000;60:33-41.

7. Baer L, Jenike MA. Personality disorders in obsessive compulsive disorder. Psychiatr Clin North Am 1992;15:803-812.

8. Black DW, Noyes R Jr, Pfohl B, Goldstein RB, Blum N. Personality disorder in obsessive-compulsive volunteers, well comparison subjects, and their first-degree relatives. Am J Psychiatry 1993;150:1226-1232.

9. Bejerot S, Ekselius L, von Knorring L. Comorbidity between obsessivecompulsive disorder (OCD) and personality disorders. Acta Psychiatr Scand 1998;97:398-402.

10. Ha TH, Youn T, Rho KS, Kim MS, Kwon JS. Symptom dimensions of obsessive-compulsive disorder and their relation to comorbid personality pathology. J Korean Neuropsychiatr Assoc 2004;43:46-53.

11. Hermesh H, Shahar A, Munitz H. Obsessive-compulsive disorder and borderline personality disorder. Am J Psychiatry 1987;144:120-122.

12. Ansell EB, Pinto A, Edelen MO, Markowitz JC, Sanislow CA, Yen S, et al. The association of personality disorders with the prospective 7-year course of anxiety disorders. Psychol Med 2011;41:1019-1028.

13. Seol SH, Kwon JS, Shin MS. Korean self-report version of the YaleBrown Obsessive-Compulsive Scale: factor structure, reliability, and validity. Psychiatry Investig 2013;10:17-25.

14. First MB, Gibbon M, Spitzer RL. Structured Clinical Interview for DSM-IV Axis II Personality Disorders. Washington DC: American Psychiatric Press; 1997.

15. Ekselius L, Lindström E,von Knorring L, Bodlund O, Kullgren G. SCID II interviews and the SCID screen questionnaire as diagnostic tools for personality disorders in DSM-III-R. Acta Psychiatr Scand 1994;90: 120-123.

16. Lee YH, Song JY. A study of the reliability and the validity of the BDI, SDS, and MMPI-D scales. Korean J Clin Psychol 1991;10:98-113.

17. Yook SP, Kim ZS. A clinical study on the Korean version of Beck Anxiety Inventory: comparative study of patient and non-patient. Korean J Clin Psychol 1997;16:185-197.

18. Lee DW, Kang UG, Lyoo IK, Jeong DU, Cho MJ, Woo JI, et al. Comorbid axis-II disorders in patients with obsessive-compulsive disorder. J Korean Neuropsychiatr Assoc 2001;40:434-442.

19. Hollander E. Managing aggressive behavior in patients with obsessivecompulsive disorder and borderline personality disorder. J Clin Psychiatry 1999;60(Suppl 15):38-44.

20. Hollander E. Obsessive-Compulsive Related Disorders. Washington DC: American Psychiatric Press; 1993.

21. Svrakic DM, McCallum K, Milan P. Developmental, structural, and clinical approach to narcissistic and antisocial personalities. Am J Psychoanal 1991;51:413-432.

22. Torres AR, Moran P, Bebbington P, Brugha T, Bhugra D, Coid JW, et al. Obsessive-compulsive disorder and personality disorder: evidence from the British National Survey of Psychiatric Morbidity 2000. Soc Psychiatry Psychiatr Epidemiol 2006;41:862-867.

23. Ricciardi JN, Baer L, Jenike MA, Fischer SC, Sholtz D, Buttolph ML. Changes in DSM-III-R axis II diagnoses following treatment of obsessive-compulsive disorder. Am J Psychiatry 1992;149:829-831. 